\title{
Constrained Control of Switched Positive Systems with Discrete and Distributed Delays
}

\author{
Jun SHEN， James LAM \\ Department of Mechanical Engineering, The University of Hong Kong, Pokfulam Road, Hong Kong \\ E-mail: junshen2009@gmail.com; james.lam@hku.hk
}

\begin{abstract}
This paper is concerned with constrained control of switched positive linear systems with discrete and distributed delays. Our goal is to design a nonnegative controller with a prescribed upper bound, such that the closed-loop system is positive and its state trajectory is located in a boundary whenever the initial condition is within the same boundary. In addition, we investigate the case when the state trajectory never reaches the boundary. It is shown that these problems are solvable via linear programming. Both continuous- and discrete-time cases are investigated in this paper. Finally, several illustrative examples are provided to show the validity of the proposed approach.
\end{abstract}

Key Words: Constrained control, Positive systems, Switched systems, Time-delay systems

\section{Introduction}

Positive systems are often utilized to capture physical systems involving quantities which are in nature nonnegative. Unlike general linear systems, the state variables of such systems are confined within a cone instead of a linear space. More specifically, the state and output of positive systems are always constrained in the nonnegative orthant whenever the input and initial condition are nonnegative. Positive systems are frequently encountered in a variety of disciplines, including systems biology, pharmacokinetics [1], ecology [2] and epidemiology. Such systems naturally arise in the modeling of heat exchange, compartmental networks [3] and genetic regulatory networks [4]. It is shown in [5] that some electrical circuits consisting of resistors, capacitors, coils, voltage and current sources can also be modeled by positive systems. Due to their broad applications, great efforts have been devoted to the analysis and synthesis of positive systems in the literature, one may refer to [6-11] and the references therein.

Constrained control, which is also related to controller design with input saturation, has been extensively studied for general linear system, one can refer to [12-14] for some recent developments. This is mainly due to the fact that real plants involve saturations on the input and system states and thus it is of importance to design controllers such that both the input and the state are confined within a prescribed boundary. The study of bounded control for positive system was initiated in $[15,16]$, where the state-feedback controller synthesis with the requirement of positivity and boundedness was solved based on linear programming. The results were further extended to discrete- and continuous-time positive linear systems with constant discrete delays in [17] and [18], respectively. However, to the best of our knowledge, the above mentioned works only investigate positive systems with constant delays and constrained control of continuoustime positive systems with time-varying delays has not been addressed to date. On the other hand, the synthesis techniques for constrained control of positive systems are quite related to the stabilization problem. Therefore, we recall some results on the stabilization of positive systems.

This work was partially supported by GRF HKU 7140/11E.
The state-feedback stabilization problem for positive systems was firstly solved in [19] in terms of linear matrix inequalities. The linear programming based state-feedback controller synthesis method was proposed in [16]. Recently, it is shown in [20] that the output-feedback stabilization problem for positive systems with single input or single output can also be solved directly by linear programming.

Motivated by the above discussion, in this paper, we address the constrained control problem for delayed positive linear systems under arbitrary switching signals. The lemmas [18, Lemmas 3,4] play a key role in the analysis of constrained control for continuous-time positive systems with constant delays. However, the technical proof for these basic lemmas in [18] has to resort to a step-by-step construction of the solution and therefore does not work for the time-varying delay case. In contrast, our approach only relies on the linearity and positivity of the system and therefore is also valid for switched positive systems timevarying discrete and distributed delays. Also note that the methods on constrained control for discrete-time positive systems with delays in [21] rely heavily on mathematical induction and hence are no longer applicable for the analysis of the continuous-time counterpart. In addition, the statefeedback controller proposed in $[17,18]$ makes use of the delayed state, which is no longer applicable when the magnitudes of the delays are unknown. In this paper, we only design a memoryless controller without delayed feedback, which also works when the delays are unknown. It is worth mentioning that the framework considered in this paper is very general, where the discrete delays are not restricted to be bounded and the switching signal can be arbitrary.

\section{Notations}

In this section, we introduce some notations on positive systems, which will be frequently used in the following sections. $\mathbb{Z}, \mathbb{N}, \mathbb{N}_{+}$and $\mathbb{R}$ denote the set of all the integers, nonnegative integers, positive integers and real numbers, respectively. All the matrices, if their dimensions are not explicitly stated, are assumed to be compatible for algebraic operations. 1 stands for a column vector with each entry equals 1. $\operatorname{diag}\left\{x_{1}, x_{2}, \ldots, x_{n}\right\}$ stands for a diagonal matrix 
with diagonal entries being $x_{1}, x_{2}, \ldots, x_{n}$. A real matrix $A \in$ $\mathbb{R}^{m \times n}$ with all of its entries nonnegative is called nonnegative matrix and is denoted by $A \succeq 0$ and $A \in \overline{\mathbb{R}}_{+}^{m \times n}$. A square matrix $A \in \mathbb{R}^{n \times n}$ with all its off-diagonal entries nonnegative is called Metzler and is denoted by $\mathbb{M}^{n}$. For two matrices $A=\left[a_{i j}\right], B=\left[b_{i j}\right] \in \mathbb{R}^{m \times n}, A \succeq B$ (respectively, $A \succ B$ ) means that $a_{i j} \geq b_{i j}$ (respectively, $a_{i j}>b_{i j}$ ) for $i=1,2, \ldots, m$ and $j=1,2, \ldots, n$.

\section{Continuous-time Case}

Consider the following continuous-time switched positive linear systems with discrete and distributed delays:

$$
\left\{\begin{aligned}
\dot{x}(t)= & A_{\sigma(t)} x(t)+A_{d \sigma(t)} x(t-d(t)) \\
& +A_{h \sigma(t)} \int_{-h(t)}^{0} x(t+s) \mathrm{d} s+B_{\sigma(t)} u(t), \quad t \geq 0, \\
x(s)= & \phi(s), \quad s \leq 0,
\end{aligned}\right.
$$

where the map $\sigma(t):[0, \infty) \rightarrow\{1,2, \ldots, N\}$ is a rightcontinuous piecewise-constant function, representing the switching signal with $N$ being the number of subsystems. $x(t) \in \mathbb{R}^{n}$ is the state vector; $u(t) \in \mathbb{R}^{m}$ is the control input; $A_{i}, A_{d i}$ and $A_{h i}$ stand for the system matrices of the $i$ th subsystem or the $i$ th mode of system (1); $\phi(\cdot)$ denotes the initial condition. In this paper, it is assumed that $0 \leq h(t) \leq h$ $(t \geq 0)$ with $h>0$ and the discrete delay $d(t) \geq 0$ is not restricted to be bounded.

Definition 1 [22] System (1) is called (internally) positive if for all initial condition $\phi(s) \succeq 0(s \leq 0)$ and input $u(t) \succeq 0$ $(t \geq 0)$, the state trajectory $x(t) \succeq 0$ for all $t \geq 0$.

Lemma 1 System (1) is positive for arbitrary switching signal $\sigma(t)$ and all delays $d(t) \geq 0,0 \leq h(t) \leq h$ if and only if for all $i=1,2, \ldots, N, A_{i}$ is Metzler, $A_{d i}, A_{h i}$ and $B_{i}$ are all nonnegative.

Proof. (Sufficiency) It suffices to prove that for any $T>0$, $x(t) \succeq 0$ for all $t \in[0, T]$. Firstly, it is easy to obtain that all the subsystems are positive by an argument similar to the proof of [23, Theorem II.2]. Then, we can prove the positivity of switched system (1) by mathematical induction. Suppose that the switching sequence over the interval $[0, T]$ is $0 \leq t_{1} \leq t_{2} \leq \ldots \leq t_{k} \leq \ldots \leq T$, for any input $u(t) \succeq 0$ and initial condition $\phi(s) \succeq 0(s \leq 0)$, it is obvious that $x(t) \succeq 0$ for $t \in\left[0, t_{1}\right)$. Due to the continuity of the state trajectory, one has $x\left(t_{1}\right) \succeq 0$. Suppose that $x(t) \succeq 0$ for $t \in\left[0, t_{k}\right]$ and $\sigma(t)=i$ for $t \in\left[t_{k}, t_{k+1}\right)$, it can be deduced that $x(t) \succeq 0$ since the $i$ th subsystem is positive and its initial condition is nonnegative by induction assumption. By mathematical induction, it follows that $x(t) \succeq 0$ for $t \in[0, T]$, which completes the sufficiency part.

(Necessity) By considering constant switching signal $\sigma(t) \equiv i(t \geq 0)$ and constant delays $d(t)=d, h(t)=h$, the necessity holds by [23, Theorem II.2].

The following proposition plays a key role in the formulation of the constrained control problem.

Proposition 1 Suppose that for all $i=1,2, \ldots, N, A_{i}$ is Metzler, $A_{d i}$ and $A_{h i}$ are all nonnegative. Then, given any $\bar{x} \succ 0$, for any initial condition $0 \preceq \phi(s) \preceq \bar{x}(s \leq 0)$, the state trajectory of system (1) with $u(t)=0$ satisfies that $x(t) \preceq \bar{x}$ $(t \geq 0)$ for arbitrary switching signal $\sigma(t)$ and all delays $d(t) \geq 0,0 \leq h(t) \leq h$ if and only if $\left(A_{i}+A_{d i}+h A_{h i}\right) \bar{x} \preceq 0$ for all $i=1,2, \ldots, N$.

Proof. (Sufficiency) Define $e(t) \triangleq \bar{x}-x(t)$, then $e(t)$ satisfies the following equation:

$$
\begin{aligned}
\dot{e}(t)= & A_{\sigma(t)} e(t)+A_{d \sigma(t)} e(t-d(t))+A_{h \sigma(t)} \int_{-h(t)}^{0} e(t+s) \mathrm{d} s \\
& -\left(A_{\sigma(t)}+A_{d \sigma(t)}+h(t) A_{h \sigma(t)}\right) \bar{x} .
\end{aligned}
$$

Note that the initial condition $e(s) \succeq 0$ since $\phi(s) \preceq \bar{x}$ for $s \leq 0$. Also note that $\left(A_{\sigma(t)}+A_{d \sigma(t)}+h(t) A_{h \sigma(t)}\right) \bar{x} \preceq$ $\left(A_{\sigma(t)}+A_{d \sigma(t)}+h A_{h \sigma(t)}\right) \bar{x} \preceq 0$, since both $A_{h \sigma(t)}$ and $\bar{x}$ are nonnegative. By regarding $-\left(A_{\sigma(t)}+A_{d \sigma(t)}+h(t) A_{h \sigma(t)}\right) \bar{x}$ as a nonnegative input, it follows that $e(t) \succeq 0$ for all $t \geq 0$ due to the positivity of the error system (2). Therefore, $x(t) \preceq \bar{x}$ for all $t \geq 0$, which completes the sufficiency part.

(Necessity) Fix $\sigma(t)=i, d(t)=d$ and $h(t)=h(t \geq 0)$, then by noting that $0 \succeq \dot{x}(0+)=\left(A+A_{d i}+h A_{h i}\right) \bar{x}$, the necessity directly follows.

Different from Proposition 1, the following proposition ensures that the state trajectory never hits the boundary $\bar{x}$.

Proposition 2 Suppose that for all $i=1,2, \ldots, N, A_{i}$ is Metzler, $A_{d i}$ and $A_{\text {hi }}$ are all nonnegative. Also assume that there exists $\bar{x} \succ 0$, such that $\left(A_{i}+A_{d i}+h A_{h i}\right) \bar{x} \prec 0$ for all $i=1,2, \ldots, N$. Then, for arbitrary switching signal $\sigma(t)$, any delays $d(t) \geq 0,0 \leq h(t) \leq h$ and any initial condition $0 \preceq \phi(s) \preceq \bar{x}(s \leq 0)$, the state trajectory of system (1) with $u(t)=0$ satisfies that $x(t) \prec \bar{x}$ for all $t>0$.

Proof. Define $e(t) \triangleq \bar{x}-x(t)$, then $e(t)$ satisfies equation (2). Since $A_{i}(i=1,2, \ldots, N)$ are Metzler matrices, one can find a sufficiently large $\mu>0$, such that $\mu I+A_{i} \succeq 0$ for all $i=1,2, \ldots, N$. Denote $U(t) \triangleq\left(\mu I+A_{\sigma(t)}\right) e(t)+A_{d \sigma(t)} e(t-$ $d(t))+A_{h \sigma(t)} \int_{-h(t)}^{0} e(t+s) \mathrm{d} s$ and we can rewrite the error system (2) as

$$
\dot{e}(t)=-\mu e(t)+U(t)-\left(A_{\sigma(t)}+A_{d \sigma(t)}+h(t) A_{h \sigma(t)}\right) \bar{x} .
$$

Then, it can be readily obtained that

$$
\begin{aligned}
e(t)= & e^{-\mu t} e(0)+\int_{0}^{t} e^{-\mu(t-\tau)} U(\tau) \mathrm{d} \tau \\
& -\int_{0}^{t} e^{-\mu(t-\tau)}\left(A_{\sigma(\tau)}+A_{d \sigma(\tau)}+h(\tau) A_{h \sigma(\tau)}\right) \bar{x} \mathrm{~d} \tau .
\end{aligned}
$$

Note that $e(t) \succeq 0$ for $t \geq 0$ by Proposition 1 and hence $U(t) \succeq 0$ for $t \geq 0$. Since $\left(A_{\sigma(t)}+A_{d \sigma(t)}+h(t) A_{h \sigma(t)}\right) \bar{x} \preceq$ $\left(A_{\sigma(t)}+A_{d \sigma(t)}+h A_{h \sigma(t)}\right) \bar{x} \prec 0$ for all $t \geq 0$, it follows that $\int_{0}^{t} e^{-\mu(t-\tau)}\left(A_{\sigma(\tau)}+A_{d \sigma(\tau)}+h(\tau) A_{h \sigma(\tau)}\right) \bar{x} \mathrm{~d} \tau \prec 0$ for $t>0$ and thus $e(t) \succ 0$ for all $t>0$. This completes the proof.

In the sequel, we focus on system (1) with a memoryless state-feedback control input $u(t)=K x(t)$. Given an upper bound $\bar{u} \succ 0$ on the controller, our goal is to find $\bar{x} \succ 0$ as well as a controller gain matrix $K$, such that the closed-loop system (1) under control input $u(t)$ is positive and satisfies that for any initial condition $0 \preceq \phi(s) \preceq \bar{x}(s \leq 0)$, it holds that the state trajectory $0 \preceq x(t) \preceq \bar{x}$ (or $0 \preceq x(t) \prec \bar{x}$ ) and the 
input $0 \preceq u(t) \preceq \bar{u}$ for all $t>0$. This synthesis problem is addressed in the following theorems.

Theorem 1 Assume that $A_{d}, A_{h} \in \overline{\mathbb{R}}_{+}^{n \times n}$. Given $u \succ 0$, for arbitrary switching signal $\sigma(t)$ and all delays $d(t) \geq 0,0 \leq$ $h(t) \leq h$, there exist $\bar{x} \succ 0$ and a feedback gain matrix $K$, such that system (1) under control input $u(t)=K x(t)$ satisfies that $0 \preceq x(t) \preceq \bar{x}$ and $0 \preceq u(t) \preceq \bar{u}$ for all $t>0$ whenever $0 \preceq$ $\phi(s) \preceq \bar{x}(s \leq 0)$ if and only if there exist a diagonal matrix $X$ with positive diagonal entries and matrix $Z \succeq 0$, such that the following linear program is feasible:

$$
\begin{aligned}
A_{i} X+B_{i} Z & \in \mathbb{M}^{n}, \\
\left(A_{i}+A_{d i}+h A_{h i}\right) X \mathbf{1}+B_{i} Z \mathbf{1} & \preceq 0, \\
Z \mathbf{1} & \preceq \bar{u}, \quad i=1,2, \ldots, N .
\end{aligned}
$$

Then, $\bar{x}$ and $K$ can be obtained as $\bar{x}=X 1$ and $K=Z X^{-1}$, respectively.

Proof. (Sufficiency) Denote $\bar{x}=X \mathbf{1}$ and $K=Z X^{-1}$, it follows from the first condition in (3) that $\left(A_{i}+B_{i} K\right) X$ is Metzler for $i=1,2, \ldots, N$. Hence, $A_{i}+B_{i} K$ is Metzler since $X$ is a diagonal matrix with positive diagonal entries, which implies the positivity of system (1) under control input $u(t)$. Constraint $\left(A_{i}+A_{d i}+h A_{h i}\right) X \mathbf{1}+B_{i} Z \mathbf{1} \preceq 0$ is equivalent to $\left(A_{i}+B_{i} K+A_{d i}+h A_{h i}\right) \bar{x} \preceq 0$, which results in $0 \preceq x(t) \preceq \bar{x}$ for $t \geq 0$ by Proposition 1 . Note that constraints $Z \mathbf{1} \preceq \bar{u}$ and $Z \succeq 0$ imply that $K \bar{x} \preceq \bar{u}$ and $K \succeq 0$, respectively, which further yields that $0 \preceq u(t)=K x(t) \preceq K \bar{x} \preceq \bar{u}$ since $0 \preceq x(t) \preceq \bar{x}$. This completes the sufficiency part.

(Necessity) Let us fix $\sigma(t)=i$ and consider constant delays $d(t)=d$ and $h(t)=h$. Note that by Proposition 1, $0 \preceq x(t) \preceq \bar{x}$ implies that $\left(A_{i}+B_{i} K+A_{d i}+h A_{h i}\right) \bar{x} \preceq 0$. Also note that $u(0)=K \bar{x} \preceq \bar{u}$. Denote $X=\operatorname{diag}\left\{\overline{x_{1}}, \overline{\overline{x_{2}}}, \ldots, \overline{x_{n}}\right\}$ where $\overline{x_{i}}$ is the $i$ th entry of vector $\bar{x}$. Then, it follows that $\left(A_{i}+B_{i} K+A_{d i}+h A_{h i}\right) X \mathbf{1} \preceq 0$ and $u(0)=K X \mathbf{1} \preceq \bar{u}$. In addition, $K \succeq 0$ can be inferred from $u(0)=K x(0) \succeq 0$ with arbitrary $0 \preceq x(0) \preceq \bar{x}$. The positivity of system (1) under control input $u(t)$ reveals that $A_{i}+B_{i} K$ is Metzler for $i=1,2, \ldots, N$. Since $X$ is a diagonal matrix with positive diagonal entries, it is obvious that $\left(A_{i}+B_{i} K\right) X$ is Metzler for $i=1,2, \ldots, N$. Denoting $Z=K X$ and summarizing the above conditions, we immediately arrive at constraints (3).

Theorem 2 Assume that $A_{d}, A_{h} \in \overline{\mathbb{R}}_{+}^{n \times n}$. Given $\bar{u} \succ 0$, for arbitrary switching signal $\sigma(t)$ and all delays $d(t) \geq 0$, $0 \leq h(t) \leq h$, there exist $\bar{x} \succ 0$ and a feedback gain matrix $K$, such that system (1) under control input $u(t)=K x(t)$ satisfies that $0 \preceq x(t) \prec \bar{x}$ and $0 \preceq u(t) \preceq \bar{u}$ for all $t>0$ whenever $0 \preceq \phi(s) \preceq \bar{x}(s \leq 0)$ if there exist a diagonal matrix $X$ with positive diagonal entries and matrix $Z \succeq 0$, such that the following linear program is feasible:

$$
\begin{aligned}
A_{i} X+B_{i} Z & \in \mathbb{M}^{n}, \\
\left(A_{i}+A_{d i}+h A_{h i}\right) X \mathbf{1}+B_{i} Z \mathbf{1} & \prec 0, \\
Z \mathbf{1} & \preceq \bar{u}, \quad i=1,2, \ldots, N .
\end{aligned}
$$

Then, $\bar{x}$ and $K$ can be obtained as $\bar{x}=X 1$ and $K=Z X^{-1}$, respectively.

Theorem 2 can be proved by following a line similar to that of Theorem 1.

\section{Discrete-time Case}

In this section, let us consider the following discretetime switched positive linear systems with discrete and distributed delays:

$$
\left\{\begin{aligned}
x(k+1)= & A_{\sigma(k)} x(k)+A_{d \sigma(k)} x(k-d(k)) \\
& +A_{h \sigma(k)} \sum_{s=1}^{h(k)} x(k-s)+B_{\sigma(k)} u(k), \quad k \in \mathbb{N}, \\
x(s)=\phi(s), \quad s \leq 0, s \in \mathbb{Z}, &
\end{aligned}\right.
$$

where the map $\sigma(k): \mathbb{N} \rightarrow\{1,2, \ldots, N\}$ stands for the switching signal with $N$ representing the number of subsystems. $x(k) \in \mathbb{R}^{n}$ denotes the state vector; $u(k) \in \mathbb{R}^{m}$ is the control input; $\phi(\cdot)$ is the initial condition. In this section, we assume that for all $k \in \mathbb{N}, h(k) \in \mathbb{N}_{+}$and $1 \leq h(k) \leq h$ with $h \in \mathbb{N}_{+}$. The delay $d(k) \geq 0$ can be unbounded. This model can be regarded as a discretized model of system (1).

Definition 2 [22] System (5) is called (internally) positive if for all initial condition $\phi(s) \succeq 0(s \leq 0, s \in \mathbb{Z})$ and input $u(k) \succeq 0(\forall k \in \mathbb{N})$, the state trajectory $x(k) \succeq 0$ for all $k \in \mathbb{N}$.

Lemma 2 System (5) is positive for arbitrary switching signal $\sigma(k)$ and all delays $d(k) \in \mathbb{N}, 1 \leq h(k) \leq h$ if and only if for all $i=1,2, \ldots, N, A_{i}, A_{d i}, A_{h i}$ and $B_{i}$ are all nonnegative.

Lemma 2 can be easily proved by mathematical induction, the details are omitted here. In order to exploit the constrained control problem for discrete-time system (5), the following two propositions are needed.

Proposition 3 Suppose that for all $i=1,2, \ldots, N, A_{i}, A_{d i}$ and $A_{h i}$ are all nonnegative. Then, given any $\bar{x} \succ 0$, for any initial condition $0 \preceq \phi(s) \preceq \bar{x}(s \leq 0, s \in \mathbb{Z})$, the state trajectory of system (5) with $u(t)=0$ satisfies that $x(k) \preceq \bar{x}$ $(k \in \mathbb{N})$ for arbitrary switching signal $\sigma(k)$ and all delays $d(k) \in \mathbb{N}, 1 \leq h(k) \leq h$ if and only if $\left(A_{i}+A_{d i}+h A_{h i}-I\right) \bar{x} \preceq$ 0 for all $i=1,2, \ldots, N$.

Proof. Let $e(k) \triangleq \bar{x}-x(k)$, and $e(k)$ satisfies the following equation:

$$
\begin{aligned}
e(k+1)= & A_{\sigma(k)} e(k)+A_{d \sigma(k)} e(k-d(k)) \\
& +A_{h \sigma(k)} \sum_{s=1}^{h(k)} e(k-s) \\
& -\left(A_{\sigma(k)}+A_{d \sigma(k)}+h(k) A_{h \sigma(k)}-I\right) \bar{x}
\end{aligned}
$$

The rest of the proof is similar to that of Proposition 1 and is therefore omitted here.

Proposition 4 Suppose that $A_{i}, A_{d i}$ and $A_{\text {hi }}$ are all nonnegative for all $i=1,2, \ldots, N$. Then, given any $\bar{x} \succ 0$, for any initial condition $0 \preceq \phi(s) \preceq \bar{x}(s \leq 0, s \in \mathbb{Z})$, the state trajectory of system (5) with $u(t)=0$ satisfies that $0 \preceq x(k) \prec$ $\bar{x}\left(k \in \mathbb{N}_{+}\right)$for arbitrary switching signal $\sigma(k)$ and all delays $d(k) \in \mathbb{N}, 1 \leq h(k) \leq h$ if and only if $\left(A_{i}+A_{d i}+h A_{h i}-I\right) \bar{x} \prec$ 0 for all $i=1,2, \ldots, N$.

Proof. (Sufficiency) Let $e(k) \triangleq \bar{x}-x(k)$, and $e(k)$ satisfies equation (6). From Proposition 3, it follows that $e(k) \succeq 0$ 
for all $k \in \mathbb{N}$. Then it is obvious that $e(k+1) \succeq-\left(A_{\sigma(k)}+\right.$ $\left.A_{d \sigma(k)}+h(k) A_{h \sigma(k)}-I\right) \bar{x} \succeq-\left(A_{\sigma(k)}+A_{d \sigma(k)}+h A_{h \sigma(k)}-\right.$ $I) \bar{x} \succ 0$ for all $k \in \mathbb{N}$, which completes the sufficiency part.

(Necessity) Let $\sigma(t) \equiv i, d(k)=d$ and $h(k)=h$, then we have $x(1)=\left(A_{i}+A_{d i}+h A_{h i}\right) \bar{x} \prec \bar{x}$, which implies that $\left(A_{i}+\right.$ $\left.A_{d i}+h A_{h i}-I\right) \bar{x} \prec 0$. This completes the necessity part.

Remark 1 It is worth mentioning that unlike the continuous-time counterpart considered in Proposition 2 , the condition $\left(A_{i}+A_{d i}+h A_{h i}-I\right) \bar{x} \prec 0$ is necessary for the discrete-time case.

In the following, we aim at designing a state-feedback controller of the form $u(k)=K x(k)$ for discrete-time switched system (5). Given a prescribed upper bound $\bar{u} \succ 0$ on the controller, we aim to find a column vector $\bar{x} \succ 0$ together with a feedback gain matrix $K$, such that system (5) under control input $u(t)$ is positive and satisfies that for any initial condition $0 \preceq \phi(s) \preceq \bar{x}, 0 \preceq x(k) \preceq \bar{x}$ (or $0 \preceq x(k) \prec \bar{x})$ and $0 \preceq u(k) \preceq \bar{u}$ holds for all $k \in \mathbb{N}_{+}$. This problem is shown to be solvable via linear programming in the following theorem.

Theorem 3 Assume that $A_{d}, A_{h} \in \overline{\mathbb{R}}_{+}^{n \times n}$. Given $\bar{u} \succ 0$, for arbitrary switching signal $\sigma(k)$ and all delays $d(k) \in \mathbb{N}$, $1 \leq h(k) \leq h$, there exist $\bar{x} \succ 0$ and a feedback gain matrix $K$, such that system (5) under control input $u(k)=K x(k)$ satisfies that $0 \preceq x(k) \preceq \bar{x}$ (respectively, $0 \preceq x(k) \prec \bar{x}$ ) and $0 \preceq u(k) \preceq \bar{u}$ for $k \in \mathbb{N}_{+}$whenever the initial condition satisfies that $0 \preceq \phi(s) \preceq \bar{x}(s \leq 0, s \in \mathbb{Z})$ if and only if there exist a diagonal matrix $X$ with positive diagonal entries and matrix $Z \succeq 0$, such that the following linear program is feasible:

$$
\begin{aligned}
A_{i} X+B_{i} Z & \succeq 0, \\
\left(A_{i}+A_{d i}+h A_{h i}-I\right) X \mathbf{1}+B_{i} Z \mathbf{1} & \preceq 0(\text { respectively, } \prec 0), \\
Z \mathbf{1} & \preceq \bar{u}, \quad i=1,2, \ldots, N .
\end{aligned}
$$

Then, $\bar{x}$ and $K$ can be obtained as $\bar{x}=X 1$ and $K=Z X^{-1}$, respectively.

\section{Illustrative Examples}

Example 1 Consider discrete-time switched system (5) with two subsystems whose system matrices are given as follows:

$A_{1}=\left[\begin{array}{ccc}0.2 & 0.2 & -0.1 \\ -0.1 & -0.4 & -0.1 \\ -0.1 & 0.1 & 0.3\end{array}\right], B_{1}=\left[\begin{array}{cc}-0.7 & 0.6 \\ 0.4 & 0.4 \\ 0.2 & 0\end{array}\right]$,

$A_{d 1}=\left[\begin{array}{ccc}0.1 & 0.2 & 0 \\ 0 & 0.3 & 0 \\ 0.1 & 0.1 & 0.2\end{array}\right], A_{h 1}=\left[\begin{array}{ccc}0.02 & 0.01 & 0 \\ 0.02 & 0 & 0.05 \\ 0.02 & 0.01 & 0\end{array}\right]$,

$A_{2}=\left[\begin{array}{ccc}0.3 & -0.2 & 0.1 \\ 0.1 & -0.5 & 0.05 \\ 0.1 & 0 & 0.2\end{array}\right], B_{2}=\left[\begin{array}{cc}-1 & 0.8 \\ -0.3 & 0.6 \\ 0.3 & -0.1\end{array}\right]$,

$A_{d 2}=\left[\begin{array}{ccc}0.05 & 0 & 0.1 \\ 0.1 & 0 & 0 \\ 0 & 0 & 0.3\end{array}\right], A_{h 2}=\left[\begin{array}{ccc}0 & 0 & 0.05 \\ 0 & 0.07 & 0.03 \\ 0 & 0 & 0.05\end{array}\right]$.

The delays are chosen as $d(k)=\sqrt{k}$ and $h(k)=$ $\lfloor 4|\sin (k)|\rfloor+1$ where $\lfloor\cdot\rfloor$ is the floor function. Given $\bar{u}=\left[\begin{array}{ll}30 & 40\end{array}\right]^{T}$ and $h=4$, we aim to design a feedback gain $K$ and find a column vector $\bar{x} \succ 0$, such that system (5) under control input $u(t)$ is positive and satisfies that $0 \preceq x(k) \prec \bar{x}$ and $0 \preceq u(k) \preceq \bar{u}$ holds for all $k \in \mathbb{N}_{+}$, whenever the initial condition $0 \preceq \phi(s) \preceq \bar{x}$. Inspired by [15], we also try to maximize the boundary, that is, $\max \mathbf{1}^{T} \bar{x}$. By $\max _{Z, X} \mathbf{1}^{T} X \mathbf{1}$ subject to the constraints in (7), we can obtain a feedback gain $K$ as

$$
K=\left[\begin{array}{lll}
0.5000 & 0.3334 & 0.0385 \\
0.2501 & 1.0000 & 0.2116
\end{array}\right]
$$

and $\bar{x}$ can be attained as $\bar{x}=[26.9692,25.7866,35.2903]^{T}$. Starting from constant initial condition $\phi(s) \equiv \bar{x}$, the trajectory of system (5) under control input $u(k)$ and randomly generated switching signal is plotted in Fig. 2. The switching sequence is depicted in Fig. 1. Note that for any other initial condition $0 \preceq \phi(s) \preceq \bar{x}(s \leq 0, s \in \mathbb{Z})$, the trajectory must be below this due to the positivity of the system. It can be observed that $0 \preceq x(k) \prec \bar{x}$ for $k \in \mathbb{N}_{+}$ from Fig. 2 and it can be verified that $u(k)=K x(k) \preceq K \bar{x}=$ $\left[\begin{array}{ll}23.4386 & 39.9982\end{array}\right]^{T} \preceq \bar{u}$.

Example 2 Let us consider continuous-time switched system (1) with two subsystems represented by the following matrices:

$$
\begin{aligned}
& A_{1}=\left[\begin{array}{ccc}
-0.5 & 0.1 & -0.5 \\
0.7 & -1 & 0.5 \\
-0.1 & 0.1 & -3
\end{array}\right], B_{1}=\left[\begin{array}{cc}
0 & 0.2 \\
-1 & -0.1 \\
-0.2 & 0.45
\end{array}\right], \\
& A_{d 1}=\left[\begin{array}{ccc}
0.1 & 0 & 0.1 \\
0.1 & 0 & 0 \\
0 & 0 & 0.4
\end{array}\right], A_{h 1}=\left[\begin{array}{ccc}
0.02 & 0.01 & 0 \\
0 & 0.02 & 0.03 \\
0.04 & 0 & 0.03
\end{array}\right], \\
& A_{2}=\left[\begin{array}{ccc}
-1 & -0.5 & 1.6 \\
0.4 & 0 & 0.8 \\
-0.06 & -0.1 & -2.5
\end{array}\right], B_{2}=\left[\begin{array}{cc}
0.1 & 0.4 \\
-1.5 & 0.5 \\
-0.1 & 0.2
\end{array}\right], \\
& A_{d 2}=\left[\begin{array}{ccc}
0.1 & 0.2 & 0.5 \\
0 & 0 & 0.1 \\
0.1 & 0.3 & 0.2
\end{array}\right], A_{h 2}=\left[\begin{array}{ccc}
0 & 0 & 0.04 \\
0.02 & 0 & 0.06 \\
0.04 & 0 & 0.05
\end{array}\right] .
\end{aligned}
$$

The delays are chosen as $d(t)=0.1 t(1+\sin t)$ and $h(t)=$ $2+\cos t$. The boundary for the input is given as $\bar{u}=$ [ $\left.\begin{array}{ll}30 & 100\end{array}\right]^{T}$ and $h=3$. One can easily check that the open-loop subsystem 2 without delays is neither positive nor stable. By $\max _{Z, X} \mathbf{1}^{T} X \mathbf{1}$ subject to the constraints in (4), a feedback gain $K$ can be attained as

$$
K=\left[\begin{array}{lll}
0.1143 & 3.0641 & 0.2499 \\
0.3571 & 2.0321 & 2.5001
\end{array}\right]
$$

and $\bar{x}$ is given by $\bar{x}=\left[\begin{array}{lll}35.4268 & 7.9695 & 6.1280\end{array}\right]^{T}$. Starting from initial condition $\phi(s) \equiv \bar{x}$, the trajectory of system (1) under control input $u(t)$ and randomly generated switching signal is depicted in Fig. 4. The corresponding switching signal is given in Fig. 3. One can observe that $0 \preceq x(t) \prec \bar{x}$ for $t>0$ from Fig. 4. It can also be verified that $u(t)=K x(t) \preceq K \bar{x}=\left[\begin{array}{ll}29.9992 & 44.1677\end{array}\right]^{T} \preceq \bar{u}$.

\section{Conclusions}

In this paper, constrained control of switched positive systems with delays has been studied. Based on linear 
programming, we have designed a state-feedback controller, which is nonnegative and within a prescribed bound, such that the closed-loop system is positive and its state trajectory lies in a boundary when the initial condition is located in the same boundary. This paper can be regarded as extensions of existing works. Different from these works, the method only need to make use of the linearity and positivity of the system and therefore is still applicable when the time delays are time-varying. As a future research direction, constrained control of switched positive systems via output feedback would be an interesting yet challenging problem.

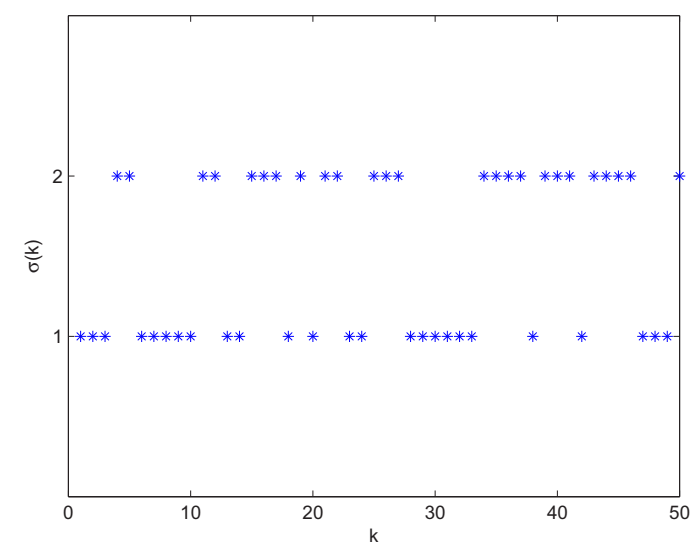

Fig. 1: Switching signal for discrete-time system (5).

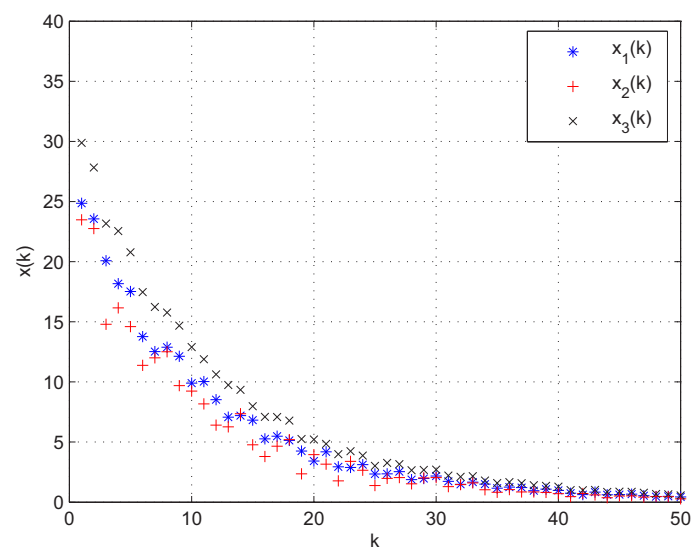

Fig. 2: State trajectory of system (5) under input $u(k)=$ $K x(k)$.

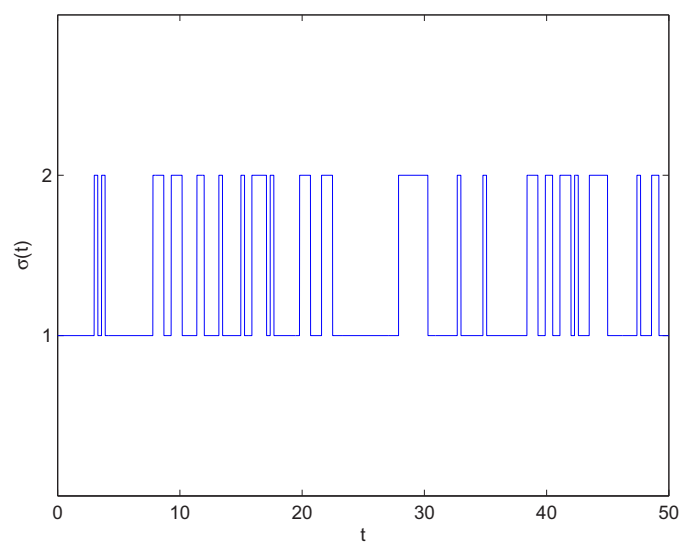

Fig. 3: Switching signal for continuous-time system (1).

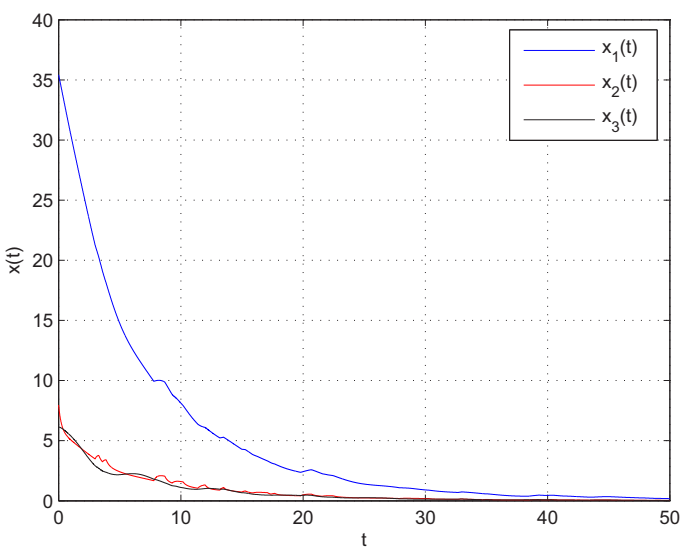

Fig. 4: State trajectory of system (1) under input $u(t)=$ $K x(t)$.

\section{References}

[1] J. Jacquez. Compartmental Analysis in Biology and Medicine. Ann Arbor, MI: Univ. Michigan Press, 1985.

[2] H. Caswell. Matrix Population Models: Construction, Analysis and Interpretation. Sunderland, MA: Sinauer Assoc., 2001.

[3] W.M. Haddad, V.S. Chellaboina, and Q. Hui. Nonnegative and Compartmental Dynamical Systems. Princeton, NJ: Princeton Univ. Press, 2010.

[4] H. de Jong. Modeling and simulation of genetic regulatory systems: a literature review. Journal of Computational Biology, 9(1):67-103, 2002.

[5] T. Kaczorek. Positive linear systems consisting of $n$ subsystems with different fractional orders. IEEE Transactions on Circuits and Systems I: Regular Papers, 58(6):1203-1210, 2011.

[6] X. Liu, W. Yu, and L. Wang. Stability analysis for continuoustime positive systems with time-varying delays. IEEE Transactions on Automatic Control, 55(4):1024-1028, 2010.

[7] X. Liu and C. Dang. Stability analysis of positive switched linear systems with delays. IEEE Transactions on Automatic Control, 56(7):1684-1690, 2011.

[8] M. Ait Rami and D. Napp. Characterization and stability of autonomous positive descriptor systems. IEEE Transations on Automatic Control, 57(10):2668-2673, 2012. 
[9] J. Shen and J. Lam. $L_{\infty}$-gain analysis for positive systems with distributed delays. Automatica, 50(1):175-179, 2014.

[10] J. Shen and J. Lam. $H_{\infty}$ model reduction for discretetime positive systems with inhomogeneous initial conditions. International Journal of Robust and Nonlinear Control, 2013. DOI: $10.1002 /$ rnc. 3075.

[11] J. Shen and J. Lam. On $\ell_{\infty}$ and $L_{\infty}$ gains for positive systems with bounded time-varying delays. International Journal of Systems Science, 2013. DOI:10.1080/00207721.2013.843217.

[12] B. Zhou, Z. Lin, and J. Lam. Semi-global stabilization of linear time-delay systems with control energy constraint. Automatica, 48(4):694-698, 2012.

[13] B. Zhou, J. Lam, and G. Duan. An ARE approach to semiglobal stabilization of discrete-time descriptor linear systems with input saturation. Systems \& Control Letters, 58(8):609616, 2009.

[14] S. Xu, G. Feng, Y. Zou, and J. Huang. Robust controller design of uncertain discrete time-delay systems with input saturation and disturbances. IEEE Transations on Automatic Control, 57(10):2604-2609, 2012.

[15] M. Ait Rami, F. Tadeo, and A. Benzaouia. Control of constrained positive discrete systems. In American Control Conference, pages 5851-5856, New York City, USA, 2007.

[16] M. Ait Rami and F. Tadeo. Controller synthesis for positive linear systems with bounded controls. IEEE Transactions on Circuits and Systems II: Express Briefs, 54(2):151-155, 2007.

[17] X. Liu, L. Wang, W. Yu, and S. Zhong. Constrained control of positive discrete-time systems with delays. IEEE Transactions on Circuits and Systems II: Express Briefs, 55(2):193-197, 2008.

[18] X. Liu. Constrained control of positive systems with delays. IEEE Transactions on Automatic Control, 54(7):1596-1600, 2009.

[19] H. Gao, J. Lam, C. Wang, and S. Xu. Control for stability and positivity: Equivalent conditions and computation. IEEE Transactions on Circuits and Systems II: Express Briefs, 52(9):540-544, 2005.

[20] M. Ait Rami. Solvability of static output-feedback stabilization for LTI positive systems. Systems \& Control Letters, 60(9):704-708, 2011.

[21] Y. Wang, L. Xu, S. Shao, D. Liu, and X. Gao. Constrained control of uncertain systems with unbounded delays. In 30th Chinese Control Conference, pages 219-223, Yantai, China, 2011.

[22] L. Farina and S. Rinaldi. Positive Linear Systems: Theory and Applications. New York: Wiley-Interscience, 2000.

[23] P.H.A. Ngoc. Stability of positive differential systems with delay. IEEE Transactions on Automatic Control, 58(1):203209, 2013. 